\title{
Myxedema coma complicated by bilateral hygromas
}

\section{Ann-Elin Meling Stokland (1), Anne Lise Dahle², Vidar Laurits Kloster3, Torbjørn Nedrebø $\emptyset^{4,5}$ and Bjørn Gunnar Nedreb $\varpi^{2,5}$}

'Department of Endocrinology, Stavanger University Hospital, Stavanger, Norway, 2Department of Internal Medicine, Haugesund Hospital, Haugesund, Norway, ${ }^{3}$ Department of Radiology, Haugesund Hospital, Haugesund, Norway, ${ }^{4}$ Department of Anaesthesia, Haraldsplass Deaconess Hospital, Bergen, Norway, and ${ }^{5}$ Department of Clinical Science, University of Bergen, Bergen, Norway
Correspondence

should be addressed

to A-E Meling Stokland

Email

ann-elin.meling.stokland@

sus.no

\section{Summary}

Myxedema coma is an important differential diagnosis in critically ill patients. Early diagnosis and treatment are paramount but challenging due to a lack of diagnostic criteria. We report a case about a patient who suffered from untreated hypothyroidism for several years. Before the correct diagnosis was made, he was admitted three times due to severe constipation. Eventually, he developed myxedema coma in connection with a urinary tract infection. The course was complicated by recurrent seizures, and neuroimaging showed bilateral hygromas. Hormone replacement therapy resulted in complete recovery and regression of hygromas. To the best of our knowledge, this is the first time hygroma is reported in association with myxedema coma.

\section{Learning points:}

- Myxedema coma is a difficult diagnosis to make due to a lack of diagnostic criteria.

- Cardinal features include hypothermia, bradycardia, gastrointestinal symptoms, pericardial/pleural effusions and affection of CNS. Anemia and hyponatremia are common.

- In case of suspected myxedema coma, neuroimaging should be a part of the evaluation in most cases.

- There is a possible association between longstanding/severe hypothyroidism and hygroma.

\section{Background}

Myxedema coma is a rare endocrine emergency with a reported incidence of $1.08 /$ million/year (1). It is most commonly reported among elderly patients, with a peak incidence in the seventh decade (1). As with other endocrine emergencies, there is often a precipitating factor, most commonly infections. Cerebrovascular accidents, congestive cardiac failure, trauma and fractures, gastrointestinal bleeding, various drugs and withdrawal of thyroid supplements may also play a role in precipitating myxedema coma (2). Diagnostic criteria for myxedema coma are lacking. A study from 2014 reported a diagnostic scoring system to predict the diagnosis, which includes affection of the CNS and gastrointestinal findings (3).
The following case highlights the consequences of hypothyroidism left untreated for several years. The patient had a typical presentation of myxedema coma, but a complicated course led to an unexpected association between clinical findings and disease. To the best of our knowledge, this is the first time hygroma is reported in association with myxedema coma.

\section{Case presentation}

A 74-year-old man was admitted three times, over a period of 2 weeks, to the surgical ward due to constipation and 
urinary retention. Prior to these admissions, he had no known chronic diseases and did not use any medication.

On examination at the last and current admission, body temperature was $35.2^{\circ} \mathrm{C}$, heart rate (HR) 53 b.p.m and blood pressure (BP) 119/80 mmHg. His abdomen was distended, and he had sparse bowel sounds. Clinical biochemistry and hematological tests showed hemoglobin (Hb) $100.8 \mathrm{~g} / \mathrm{L}$ (133-177 g/L), mean corpuscular volume $104 \mathrm{fL}$ (82-98 fL), white blood cell count (WBC) $8.710^{9} / \mathrm{L}$ (3.5-8.8 109/L), sodium $131 \mathrm{mmol} / \mathrm{L}(137-145 \mathrm{mmol} / \mathrm{L})$, potassium $3.9 \mathrm{mmol} / \mathrm{L}(3.5-5.1 \mathrm{mmol} / \mathrm{L})$, total-calcium $2.31 \mathrm{mmol} / \mathrm{L}(2.15-2.51 \mathrm{mmol} / \mathrm{L})$, creatinine $91 \mathrm{umol} / \mathrm{L}$ (60-105 umol/L), C-reactive protein (CRP) $20 \mathrm{mg} / \mathrm{L}$ $(<5 \mathrm{mg} / \mathrm{L})$ and albumin $33 \mathrm{~g} / \mathrm{L}(34-45 \mathrm{~g} / \mathrm{L})$. CT scan of the abdomen ruled out ileus. Treatment was started for constipation and suspected urinary tract infection.

After admission, he was intermittently confused and had persistent hypothermia with a temperature between 34 and $35.5^{\circ} \mathrm{C}$. His serum sodium level gradually decreased to $126 \mathrm{mmol} / \mathrm{L}$. At this point, a medical consultation was requested. Chronic mild hyponatremia over the last 3 years was noted. In the last couple of days, he had received isotonic fluids intravenously (IV), which only resulted in a further decrease in serum sodium level. Urine osmolality was $368 \mathrm{mOsmol} / \mathrm{kg}$ (300-900) and urine sodium concentration $128 \mathrm{mmol} / \mathrm{L}$. The patient reported gradually increased fatigue, lethargy, and memory and hair loss over the last 2 years. Physical examination revealed alopecia, dry skin, puffiness of the face, hoarse voice, bradycardia and hypothermia. Hypothyroidism with the development of myxedema coma was suspected.

\section{Investigation and treatment}

Supplementary blood tests confirmed thyroid-stimulating hormone (TSH) to be $94 \mathrm{mU} / \mathrm{L}(0.35-4.9)$, free thyroxine (FT4) $<6 \mathrm{pmol} / \mathrm{L}$ (9-19) and free triiodothyronine (FT3) $<1.5 \mathrm{pmol} / \mathrm{L}$ (2.6-5.7). Thyroidperoxidase antibodies (anti-TPO) was 1502 international units/milliliter (IU/ $\mathrm{mL})(<60)$, anti-thyroglobulin was $15955 \mathrm{IU} / \mathrm{mL}$, and cortisol was $338 \mathrm{nmol} / \mathrm{L}(101-536)$ at 08:00 h. Upon closer examination, he had taken blood tests 7 years earlier that revealed TSH to be $234 \mathrm{mU} / \mathrm{L}$, FT4 to be $8 \mathrm{pmol} / \mathrm{L}, \mathrm{FT} 3$ to be $2.8 \mathrm{pmol} / \mathrm{L}$, but the test result was not followed up, and he was left untreated. Levothyroxine was not available for i.v. administration at the time. Therefore, oral therapy was given; the first day at a dose of $100 \mu \mathrm{g}$, and $50 \mu \mathrm{g}$ daily from day two. In addition, he received $10 \mu \mathrm{g}$ of triiodothyronine every 12th hour. Glucocorticoid coverage was not given due to normal cortisol levels, including a normal cosyntropin test. The first day after the thyroid hormone treatment was started, the patient was feeling well but had occasional episodes of disorientation. Four days later, he was suddenly found with reduced consciousness and had several generalized seizures. At this time, his serum sodium level was stable at $128 \mathrm{mmol} / \mathrm{L}$, and the seizures were not related to hyponatremia. Serum glucose was $5.6 \mathrm{mmol} / \mathrm{L}$, $\mathrm{Hb}$ levels had decreased to $69.0 \mathrm{~g} / \mathrm{L}$. Pituitary-thyroid axis was slightly better, TSH was $56 \mathrm{mU} / \mathrm{L}$, but FT4 was still under the detection limit, and the patient had persistent hypothermia and bradycardia. CT scan of the brain was performed immediately and showed bilateral hygromas. MRI the following day confirmed major bilateral hygromas without detectable intracerebral pathology (Fig. 1). Prior to admission, the patient had long-lasting reduced cognition and developed an atactic gait, but no history of head trauma. Supplementary CT of thorax and abdomen showed no signs of ongoing bleeding but moderate amounts of pleural effusion and small amounts of pericardial effusion. Echocardiography showed normal ejection fraction and

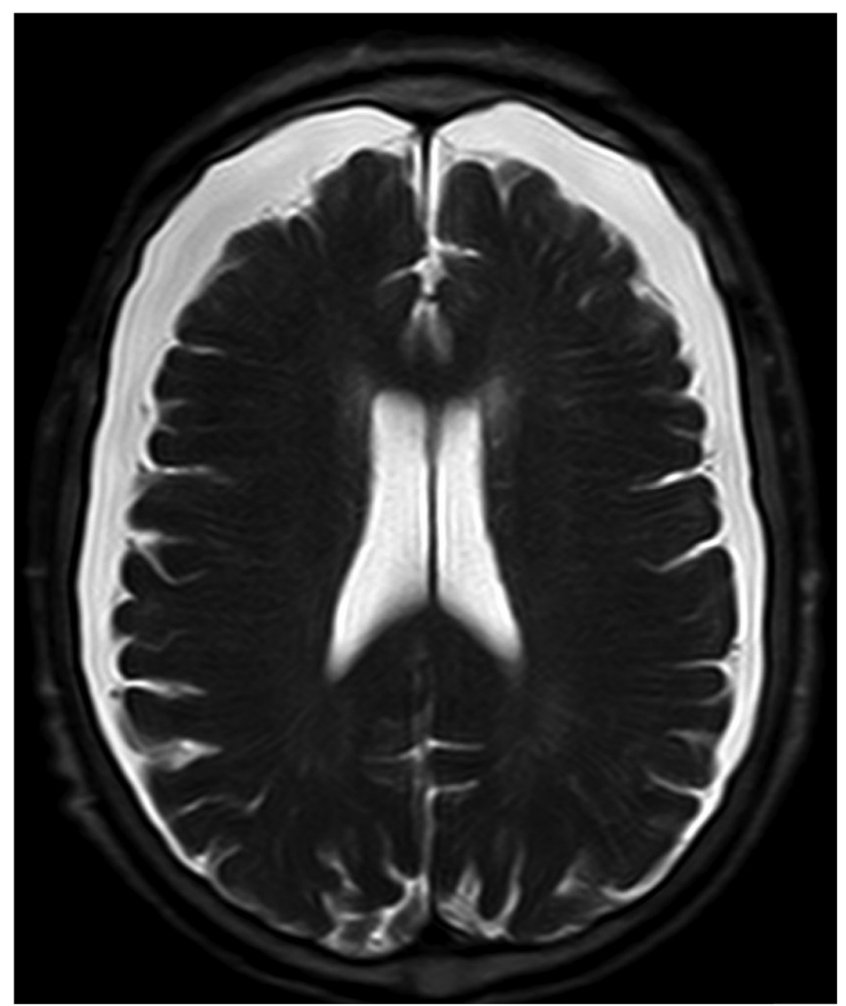

Figure 1

First MRI. The next day, after CT scan was preformed, axial T2W_TSE 5 $\mathrm{mm}$ sequence showed accumulation of fluid in the subdural space, confirming bilateral hygromas along the supratentorial cerebral convexities. Measuring 11-12 mm and containing clear fluid. The intracranial spaces are a bit compressed, but there are no signs of herniation. 
confirmed small amounts of pericardial fluid. Treatment with oral thyroxine and triiodothyronine was continued in the same dosage, and glucocorticoid coverage was added intravenously (hydrocortisone $100 \mathrm{mg} \times 4$ ), also due to the possibility of coexistent Hashimoto encephalopathy. Regarding hyponatremia, no specific measures were taken. The patient received a blood transfusion on one occasion, but thereafter his hemoglobin and serum sodium level gradually increased along with thyroid substitution therapy. Levothyroxine was not available for i.v. administration until after 1 week of treatment and was only given for 2 days. The patient was observed in the intensive care unit (ICU) and had new episodes with seizures. Between the seizures, he was mostly coherent. During the course, respiration and circulation were stable, and he was transferred from ICU to the medical ward after 2 days.

\section{Outcome and follow-up}

The patient's condition gradually improved thereafter, without new seizures. Blood tests showed gradual improvements with rising hemoglobin and serum sodium and decreasing TSH levels. Heart rate and the temperature returned to normal after a few days. The patient was discharged after 3 weeks, and an MRI before discharge found both hygromas to be reduced (Fig. 2). At follow-up in the outpatient clinic 2 months later, the pituitary-thyroid axis was normal, the patient was asymptomatic without any sequela and had even got his hair back. Control MRI 4 months after admission revealed complete regression of hygromas (Fig. 3).

\section{Discussion}

Neurological symptoms are frequent in myxedema coma. Manifestations may range from alteration of mental status with slowness, decreased concentration and lethargy, headache, cranial nerve palsies, hoarseness, myopathy, neuropathy, reflex changes, ataxia and psychotic episodes (4). Seizures are a more unusual presentation and have been associated with poor prognosis. In this case, the patient had recurrent seizures 4 days after the thyroid hormone replacement was initiated. Theoretically, severe hypothyroidism can precipitate seizure activity due to cerebral edema secondary to the expansion of the extracellular fluid volume (5). In addition to the generalized depression of cerebral function, factors like hyponatremia, hypoglycemia, hypoxemia, and reduced cerebral blood flow can precipitate focal or generalized seizures and worsen

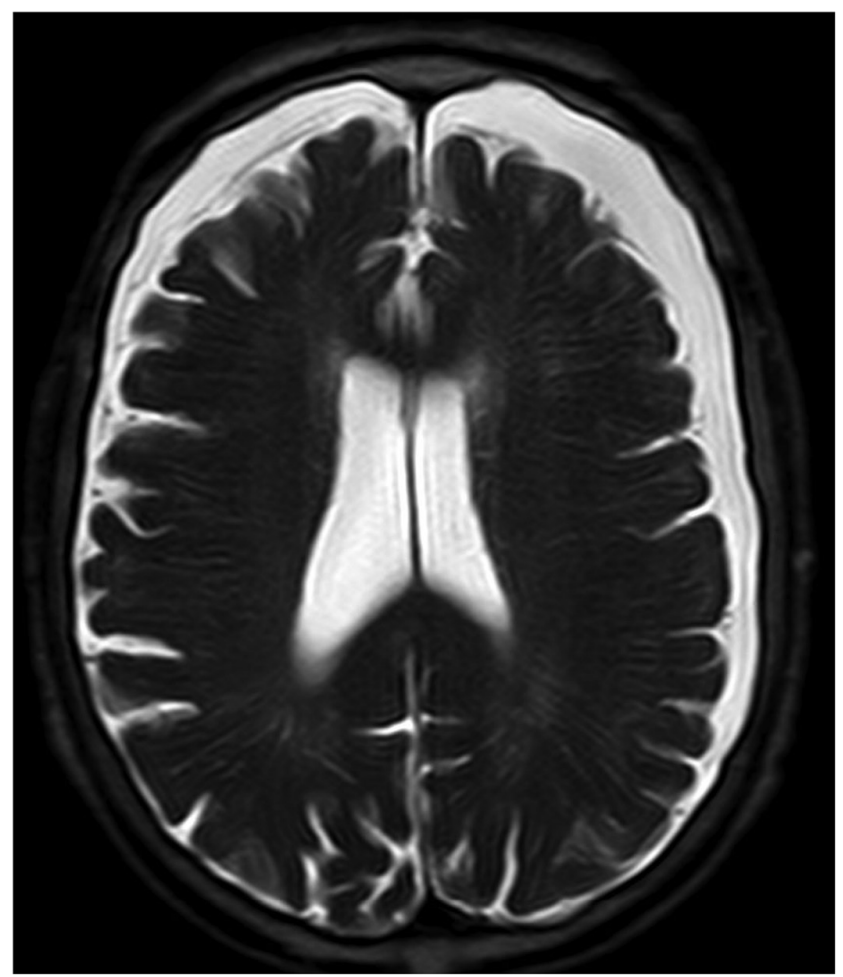

Figure 2

MRI 16 days later. Improvement of intracranial spaces and partial resorption of right sided hygroma, now measuring $8 \mathrm{~mm}$ compared to 12 $\mathrm{mm}$ earlier. Along the supratentorial cerebral convexities on the left side, there is almost the same amount of hygroma.

the level of consciousness (2). Our patient had chronic and moderate hyponatremia that was unlikely to explain the seizures. At the time of presentation, his serum sodium level had increased from 126 to $128 \mathrm{mmol} / \mathrm{L}$ after isotonic fluids were discontinued and thyroid substitution therapy was started. In addition, his blood sugar was normal, and he was not hypoxic or hypotensive. Surprisingly, he had bilateral hygromas found on CT and MRI. A subdural hygroma is an accumulation of fluid in the subdural space. It may be the result of tears, or insufficiency, in the arachnoid membrane that allows cerebrospinal fluid (CSF) to enter the subdural space, producing an extracerebral fluid collection with signal intensities identical to CSF on all imaging parameters (6). Two criteria must be present for a hygroma to occur: sufficient subdural space and separation in the dural border cell (DBC) layer. Separation of the DBC layer is usually caused by trauma, however, it is reported to occur after surgery, dehydration, artificial brain shrinkage, or excessive CSF drainage (7). Our patient had no trauma in the medical history. It is, therefore, conceivable that the hygromas occurred secondary to severe hypothyroidism, which has, to our knowledge, never been described before in the literature. The mechanism behind this is uncertain but 


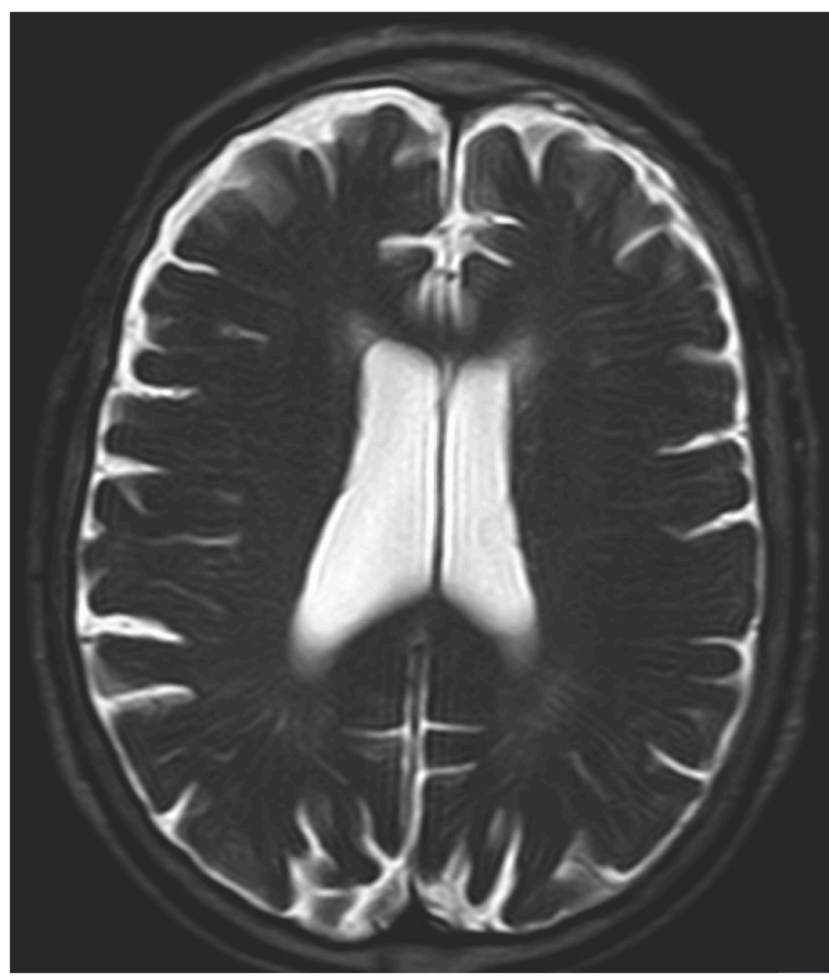

Figure 3

MRI 4 months later. The same axial T2W_TSE $5 \mathrm{~mm}$ sequence shows, 4 months later, almost no accumulation of fluid in the subdural space. Only 1-2 mm of fluid which is considered to be normal. Clear fluid in the subdural compartment.

could be related to increased permeability, disrupted water homeostasis and changes in circulatory hemodynamics. Chambers et al. examined the effect of thyroid status on brain volumes; hypothyroidism was associated with modest reductions in cerebellar and pallidum volume when controlling for overall head size (8). Our patient had been suffering from untreated hypothyroidism for several years, and it is reasonable to imagine that this has led to structural changes. Hashimoto's encephalopathy was considered as a differential diagnosis, but these patients usually have subclinical or mild overt thyroid disease and normal MRI or with non-specific abnormalities.

The treatment of myxedema coma is controversial and largely based on expert opinion and case reports. The dose and route of administration of thyroxine, and whether to use liothyronine, are still being debated. The American Thyroid Association guidelines recommend i.v. thyroid hormone replacement, with a loading dose of 200-400 $\mu \mathrm{g}$ thyroxine and thereafter a lower replacement dose of $1.6 \mu \mathrm{g} /$ $\mathrm{kg}$ body weight, reduced to $75 \%$ as long as administered intravenously. Further, the guidelines suggest that i.v. liothyronine may be given in addition to levothyroxine, in a loading dose of 5-20 $\mu \mathrm{g}$ followed by a maintenance dose of $2.5-10 \mu$ g every $8 \mathrm{~h}(9)$. However, in many countries, i.v. thyroid hormone replacement is not available. Our case is an example of successful treatment with oral combination therapy. Previous case reports are in agreement with our findings (10). In this particular case, it is conceivable that it was beneficial to treat with liothyronine as the patient had cerebral symptoms and an absence of cardiovascular complications. Liothyronine is an active hormone, which quickly reaches its peak level and has a beneficial effect on neuropsychiatric symptoms because it can cross the bloodbrain barrier (10). At the same time, one can argue that the dose of levothyroxine given was too small, especially considering oral treatment and persisting symptoms and findings. Considering the patient's age and the possibility of coronary artery disease, one can also argue to be careful with liothyronine treatment.

Mortality from myxedema comais high. In a nationwide database from Japan, in-hospital mortality was $29.4 \%$. In that study, patients treated with steroids, catecholamines, and mechanical ventilation showed significantly higher mortality rates than patients without these treatments (1). This may be related to the fact that patients receiving this type of treatment have a more serious condition. Another theory is that steroids and dopamine provoke iatrogenic hypothyroidism, which makes it paradoxical that steroids are recommended in the treatment of myxedema coma. Initially, we chose to withhold steroids due to normal cortisol and low T3 levels.

To the best of our knowledge, this is the first time hygroma is reported in association with myxedema coma. The underlying pathogenetic mechanism is uncertain but may be related to hypothyroidism-induced changes in permeability, water-homeostasis, hemodynamic changes and brain volume. After less than 3 weeks of treatment, the hygroma was declining and resulted in complete regression after 4 months.

\section{Declaration of interest}

The authors declare that there is no conflict of interest that could be perceived as prejudicing the impartiality of the research reported.

\section{Funding}

This research did not receive any specific grant from any funding agency in the public, commercial or not-for-profit sector.

\section{Patient consent}

Informed consent has been obtained from the patient for publication of the case report and accompanying images. 


\section{Author contribution statement}

Ann-Elin Meling Stokland wrote the frist draft of the manuscript which was reviewd by Bjørn Gunnar Nedrebø, Torbjørn Nedrebø, Vidar L Kloster and Anne Lise Dahle. All authors approved the final version of the manuscript. Bjørn Gunnar Nedrebø and Anne Lise Dahle were involved in patients management.

\section{References}

1 Ono Y, Ono S, Yasunaga H, Matsui H, Fushimi K \& Tanaka Y. Clinical characteristics and outcomes of myxedema coma: analysis of a national inpatient database in Japan. Journal of Epidemiology 201727 117-122. (https://doi.org/10.1016/j.je.2016.04.002)

2 Mathew V, Misgar RA, Ghosh S, Mukhopadhyay P, Roychowdhury P, Pandit K, Mukhopadhyay S \& Chowdhury S. Myxedema coma: a new look into an old crisis. Journal of Thyroid Research 20112011493462. (https://doi.org/10.4061/2011/493462)

3 Popoveniuc G, Chandra T, Sud A, Sharma M, Blackman MR, Burman KD, Mete M, Desale S \& Wartofsky L. A diagnostic scoring system for myxedema coma. Endocrine Practice 201420 808-817. (https://doi.org/10.4158/EP13460.OR)

4 Gunatilake SSC \& Bulugahapitiya U. Myxedema crisis presenting with seizures: a rare life-threatening presentation-a case report and review of the literature. Case Reports in Endocrinology 201720174285457. (https://doi.org/10.1155/2017/4285457)

5 Evans EC. Neurologic complications of myxedema: convulsions. Annals of Internal Medicine 196052 434-444. (https://doi. org/10.7326/0003-4819-52-2-434)

6 McCluney KW, Yeakley JW, Fenstermacher MJ, Baird SH \& Bonmati CM. Subdural hygroma versus atrophy on MR brain scans: 'the cortical vein sign'. American Journal of Neuroradiology 199213 1335-1339.

7 Lee KS. History of chronic subdural hematoma. Korean Journal of Neurotrauma 201511 27-34. (https://doi.org/10.13004/ kjnt.2015.11.2.27)

8 Chambers T, Anney R, Taylor PN, Teumer A, Peeters RP, Medici M, Caseras X \& Rees DA. Effects of thyroid status on regional brain volumes: a diagnostic and genetic imaging study in UK Biobank. Journal of Clinical Endocrinology and Metabolism 2021106 688-696. (https://doi.org/10.1210/clinem/dgaa903)

9 Jonklaas J, Bianco AC, Bauer AJ, Burman KD, Cappola AR, Celi FS, Cooper DS, Kim BW, Peeters RP, Rosenthal MS, et al. Guidelines for the treatment of hypothyroidism: prepared by the American Thyroid Association task force on thyroid hormone replacement. Thyroid 2014 24 1670-1751. (https://doi.org/10.1089/thy.2014.0028)

10 Ueda K, Kiyota A, Tsuchida M, Okazaki M \& Ozaki N. Successful treatment of myxedema coma with a combination of levothyroxine and liothyronine. Endocrine Journal 201966 469-474. (https://doi. org/10.1507/endocrj.EJ18-0469)

Received in final form 19 May 202

Accepted 25 May 2021 